\title{
Effect of a surface shear layer on gravity and gravity-capillary waves of permanent form
}

\author{
By F. A. MILINAZZO† AND P. G. SAFFMAN \\ Applied Mathematics 217-50, California Institute of Technology, Pasadena, CA 91125, USA
}

(Received 30 August 1989)

Calculations are carried out of the shape of gravity and gravity-capillary waves on deep water in the presence of a thin sheet of uniform vorticity which models the effect of a wind drift layer. The dependence of the fluid speed at the wave crest is determined and compared for gravity waves with the theory of Banner \& Phillips (1974). It is found that this theory underestimates the retardation due to drift and tendency to break. The retardation disappears when capillary forces are significant, but in this case it is found that there can be a significant alteration of the wave shape.

\section{Introduction}

We consider in this work the properties of two-dimensional, periodic water waves of permanent form on deep water when a thin shear layer is supposed to be present, caused for example by wind stress. The layer is supposed to have an average depth $\Delta$, and contain a constant uniform vorticity $-\Omega$. The approximation of constant vorticity is made in order to simplify the analysis. In principle, an arbitrary vorticity distribution can be employed, but the calculations are then much harder (cf. Moore \& Saffman 1982). Below the shear layer, the flow is assumed to be irrotational. Effects on the waves of viscosity and the air motion after the shear layer has been set up are supposed negligible.

To formulate the problem mathematically, we move to a coordinate system moving with the wave in which the flow is steady. The problem is to find surfaces with spatial wavelength $\lambda, y=H_{1}(x), y=H_{2}(x)$, with $\bar{H}_{1}=0, \bar{H}_{2}=-\Delta$, and stream functions $\Psi_{1}(x, y), H_{2}<y<H_{1}$, and $\Psi_{2}(x, y),-\infty<y<H_{2}$, where

$$
\begin{aligned}
& \nabla^{2} \Psi_{1}=\Omega ; \quad \nabla^{2} \Psi_{2}=0 \\
& \Psi_{1}=\text { const. }, \quad y=H_{1} ; \quad \Psi_{1}=\text { const. }, \quad y=H_{2} ; \quad \Psi_{2}=\text { const. }, \quad y=H_{2} \\
& \nabla \Psi_{1}=\nabla \Psi_{2}, \quad y=H_{2} ; \\
& p+T / R=\text { const., } \quad y=H_{1} \text {; } \\
& \Psi_{2} \sim-c y \text { as } y \rightarrow \infty \text {; }
\end{aligned}
$$

where $c$ is the wave speed, $p$ is the pressure, $T$ is the surface tension and $R$ is the radius of curvature of the surface. See figure 1 .

For the case when surface tension is neglected, Simmen \& Saffman (1985) considered the case of an infinitely deep shear layer, i.e. $\Delta=\infty$. Teles da Silva \& Peregrine (1988) found solutions for finite depth, where the bottom surface is a rigid

† Permanent address: Royal Roads Military College, Victoria, BC, Canada. 


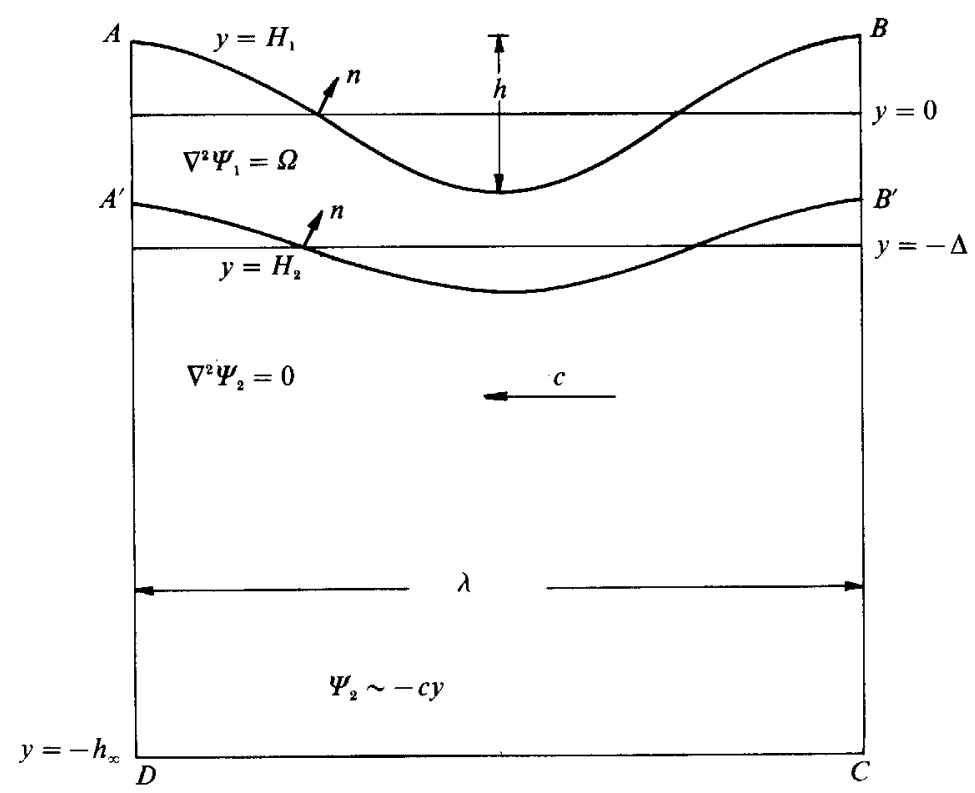

Figure 1. Sketch of the geometry.

plane, i.e. $H_{2}=-\Delta$. We are not aware of any computed or analytic solutions for the mathematical problem just formulated, especially for the case $\Delta \ll \lambda$. Banner $\&$ Phillips (1974, herein referred to as BP) examined this case by ingeniously applying conservation of vorticity to a one-dimensional description of the shear layer together with the Levi-Cività result (see paragraph below containing equation $(2.10)$ ) to obtain the relation

$$
\left(u_{\mathrm{s}}\right)_{\mathrm{crest}}=-\left[\left(c-U_{0}\right)^{2}-q_{0}\left(2 c-q_{0}\right)\right]^{\frac{1}{2}},
$$

where $u_{\mathrm{s}}$ is the speed of the fluid at the surface in the wave-fixed coordinate system and the value at the crest is $\partial \Psi_{1} /\left.\partial y\right|_{y=\max H_{1}}, U_{0}$ is the maximum forward orbital velocity in the irrotational part of the wave, i.e. $c+\partial \Psi_{2} /\left.\partial y\right|_{y=\max H_{2}}$, and $q_{0}(\approx \Omega \Delta)$ is the surface drift at the mean level of the surface. There is some ambiguity in the definition of $q_{0}$, and we shall follow the BP definition, which is $q_{0}=\partial \Psi_{1} /\left.\partial n\right|_{y=0}+c$.

$\mathrm{BP}$ argue that the limiting wave is attained, and incipient breaking occurs, when a stagnation point occurs on the surface. According to their analysis of the shear layer, this occurs first at the crest and the condition for incipient breaking follows from (1.6);

$$
q_{0}\left(2 c-q_{0}\right)=\left(c-U_{0}\right)^{2} .
$$

They also deduce from Bernoulli's equation that

$$
\max H_{1}=\frac{\left(c-q_{0}\right)^{2}}{2 g}
$$

Thus drift can reduce significantly the maximum elevation for breaking. Note that the theory does not give the limiting value for $h$, where $h=\max H_{1}-\min H_{1}$ is the vertical distance between crest and trough on the surface and will be called the wave height, as this requires knowledge of the speed in the wave troughs. Also their comment that in a rotational flow a stagnation point at the crest need not be associated with a discontinuity in surface slope is not correct. The angle in the cusp 
may, however, look more like $90^{\circ}$ than $120^{\circ}$ if $\Omega^{2} \gg g / \lambda$ and the resolution is not fine (Broadbent \& Moore 1985).

One purpose of the present study is to investigate the range of $\Delta$ for the $\mathrm{BP}$ argument to hold, i.e. to consider how thin the shear layer should be given $q_{0}$ or $\Omega \Delta$, and obtain information about the limiting wave height (and not just the elevation). A further purpose is to incorporate surface tension and determine its effect on the conditions for a stagnation point to occur on the surface and the shape of the waves. We have been unable to extend the BP argument to include surface tension effects because the Levi-Cività result does not then hold. Their comment about a stagnation point not necessarily being associated with a singularity is, however, correct in the presence of surface tension.

Also, we wish to establish formulations of the steady waves that will enable a stability investigation to be carried out. (For finite depth, a stability calculation to both two- and three-dimensional disturbances has been performed by Okamura \& Oikawa 1989.) We plan to report on a study of the stability of waves with a thin shear layer in subsequent work.

\section{Fourier series formulation}

Solutions for steep waves are not currently obtainable by analytic methods, and numerical approaches are necessary. In this work we use truncated Fourier series (Dalrymple 1974) to represent the stream functions and the fluid surfaces. This approach gives accurate solutions quickly with little work if the wave height $h$ is not too large and allows us to establish the number of independent parameters for the problem. Unfortunately if the waves are too steep, the method fails and then other formulations such as boundary integral representations or spectral solutions of the partial differential equations are more appropriate. These will be described in subsequent work.

We take, restricting attention to waves symmetrical about crests and troughs, $\dagger$ and putting $\lambda=2 \pi$,

$$
\begin{gathered}
H_{1}=\sum_{1}^{N} c_{n} \cos n x \\
\Psi_{1}=\frac{1}{2} \Omega y^{2}-\hat{c} y+\hat{Q}+\sum_{1}^{N} \cos n x\left(a_{n} \mathrm{e}^{n y}+b_{n} \mathrm{e}^{-n y}\right), \\
H_{2}=-\Delta+\sum_{1}^{N} d_{n} \cos n x \\
\Psi_{2}=-c y+Q+\sum_{1}^{N} f_{n} \mathrm{e}^{n y} \cos n x .
\end{gathered}
$$

The boundary conditions are

$$
\begin{gathered}
\Psi_{1}=0 \quad \text { on } y=H_{1}, \\
\frac{1}{2}\left(\nabla \Phi_{1}\right)^{2}+g H_{1}-\frac{T H_{1}^{\prime \prime}}{\left(1+\left(H_{1}^{\prime}\right)^{2}\right)^{\frac{3}{2}}}=B_{1} \quad \text { on } y=H_{1}, \\
\Psi_{1}=\Psi_{2}=K \text { on } y=H_{2} \\
\partial \Phi_{1} / \partial y=\partial \Psi_{2} / \partial y \text { on } y=H_{2} .
\end{gathered}
$$

+ There is considerable interest in the existence of non-symmetrical waves of permanent form. Zufiria (1987) demonstrated their existence in the absence of a shear layer for sufficiently steep gravity waves. It is hoped to study in future work the possibility of symmetry breaking in the presence of a shear layer. 
We suppose that $\Delta$ and $\Omega$ are given. Note that in (2.6) we take the pressure in the air above the upper surface to be zero.

The unknowns are $\hat{c}, \hat{Q}, c, Q, B_{1}, K$, plus $5 N$ Fourier coefficients, giving $5 N+6$ in all. Equating to zero the coefficients of $\cos n x(n=0,1, \ldots, N)$ in the boundary conditions gives $5 N+5$ equations. The last equation comes from imposing the wave height

$$
h=\sum_{1}^{N} c_{n}\left(1-(-1)^{n}\right) .
$$

This approach fails owing to divergence of the series for $\Psi_{1}$ and/or $\Psi_{2}$, even when the flow is completely free of singularities and the series for $H_{1}$ and $H_{2}$ are convergent. This occurs (Saffman \& Yuen 1982) because the analytic continuation of $\Psi_{2}$ into the shear layer develops singularities below $y=\max H_{2}$, and when the analytic continuation of $\Psi_{1}$ develops singularities below $y=\max H_{1}$ or above $y=\min H_{2}$, in which case expansions of the forms (2.2) and (2.4) are inappropriate. $\dagger$ It also fails if the surfaces become multivalued, i.e. overhanging waves develop.

The accuracy of the calculations was checked by comparing solutions obtained using 25 Fourier modes to those obtained using 50 Fourier modes. For the results presented here the first 25 Fourier modes agreed to at least 4 decimal places. It should be emphasized that this amounts to a check on the truncation error of the method of solution. The wave steepness at which the series start to diverge can be determined by monitoring the decay of the coefficients of the stream function. In some cases, this was done to determine the validity of a solution. In most cases, $H_{1}$ and $H_{2}$ were plotted and irregularities depending upon truncation in these curves were taken as an indication that the corresponding solution was suspect. We did not attempt to find accurately the critical values of $h$ at which the expansions diverged as this is not a physically significant quantity. The plotted results are conservative; the solutions might converge for values of $h / \lambda$ larger than those shown.

Unfortunately, a check on the accuracy of the solutions for gravity waves is not provided by the Levi-Cività result that the velocity on the lower interface where $H_{2}=-\Delta$ is equal to the wave speed, i.e. $\partial \Psi_{1,2} /\left.\partial n\right|_{y=-\Delta}=-c$. BP claim that the argument for the irrotational flow still holds in the presence of the shear layer, but this is not obvious. The result that we obtain (see the Appendix for details) is

$$
B_{2}=\frac{1}{2} c^{2}=\frac{1}{2 \lambda} \int_{H_{2}}|\nabla \Psi|^{2} \mathrm{~d} x,
$$

where $B_{2}$ is the Bernoulli constant on the interface $y=H_{2}$. The Levi-Cività result, $|\nabla \Psi|=c$ at the mean level $y=-\Delta$ holds only if $p-g \Delta=0$ at the mean level, or

$$
\int_{H_{2}}|\nabla \Psi|^{2} \mathrm{~d} x=\lambda|\nabla \Psi|_{y=-\Delta}^{2},
$$

and we see no reason why this is satisfied exactly. It may, however, be small, since the derivative of $p+g y$ normal to the streamlines is zero at a point of inflexion, and points on the mean level of a streamline can be expected to be not too far from points of inflexion. In fact, our computations suggest that $c-|\nabla \Psi|_{y=-\Delta}$ is of order $\Delta^{2} h^{2}$. The error in the BP theory due to use of the Levi-Cività relation should therefore not matter until the wave is steep.

$\dagger$ Compare the failure of the Taylor series for $\left(1+x^{2}\right)^{-1}$ to converge for $x>1$ owing to the existence of a non-real singularity. 


\section{Results}

In figure 2 we show plots of $\left(u_{\mathrm{s}}\right)_{\text {crest }} v s . h / \lambda$ for values of $\Omega \Delta$ from 0 to 0.5 , for four values of $\Delta$ and $T=0$, and compare them with the BP prediction of equation (1.6) evaluated using the calculated values of the variables. We have taken $g=1, \lambda=2 \pi$, and normalized the velocities on $C_{0}^{*}=\left(g^{*} \lambda^{*} / 2 \pi\right)^{\frac{1}{2}}$. The relation between dimensional quantities, denoted by an asterisk, and the dimensionless variables is as follows. $\Delta^{*}=\Delta \lambda^{*} / 2 \pi, h^{*}=h \lambda^{*} / 2 \pi, c^{*}=c\left(g^{*} \lambda^{*} / 2 \pi\right)^{\frac{1}{2}}, u_{\mathrm{s}}^{*}=u_{\mathrm{s}}\left(g^{*} \lambda^{*} / 2 \pi\right)^{\frac{1}{2}}, T^{*}=T g^{*} \lambda^{* 2} / 4 \pi^{2}$, $\Omega^{*}=\Omega\left(2 \pi g^{*} / \lambda^{*}\right)^{\frac{1}{2}}, \Omega^{*} \Delta^{*}=\Omega \Delta\left(g^{*} \lambda^{*} / 2 \pi\right)^{\frac{1}{2}}$.

The vorticity $\Omega$ can be either positive or negative, but since the equations are invariant under the transformation $c \rightarrow-c, \Omega \rightarrow-\Omega$, it is sufficient to restrict attention to positive $\Omega$ provided one allows for negative wave speeds $c$. There are three classes of waves depending upon the value of $c$ as $h$ and $\Omega \Delta \rightarrow 0$. The three values are $C_{0},-C_{0}, 0$. The $\mathrm{BP}$ case corresponds to the limit $c=C_{0}$, and this is the case we concentrate on, although the others are of interest. The results show that the drift layer significantly reduces the speed of the fluid at the crest and increases the tendency to breaking but the effect is greater than that predicted by BP, especially if $\Delta$ is large. The BP hypotheses are likely to be more accurate, the smaller the value of $\Delta$.

The dependence of $\left(u_{\mathrm{s}}\right)_{\text {crest }}$ on $\Delta$, for $h / \lambda=0.05, T=0$ and values of $\Omega \Delta$ is shown in figure 3. It can be seen that the dependence on $\Delta$ is weak, and that the main dependence is upon the drift velocity. We found from our computations that the value of $q_{0}$ as defined in $\S 1$ was very close to $\Omega \Delta$, so that $\Omega \Delta$ is a good measure of the surface drift velocity. The calculation breaks down when $h / \lambda$ becomes too large, for the reasons explained in $\$ 2$. The stronger the shear layer, the smaller the wave height at which the method fails.

Figure 4 shows the effect of shear on the profile of a gravity wave. A gravity wave of wave height $h / \lambda=0.062$ and a wave of the same height with drift $\Omega \Delta=0.456$, $\boldsymbol{\Delta}=\mathbf{0 . 4}$ are plotted. The steepening of the wave with shear is quite noticeable.

The result of a calculation for non-zero surface tension is shown in figure 5 . We plot the free surface and the interface for a case with $\Omega=1.0, \Delta=0.05, T=0.2$, and $h / \lambda=0.026$. Also shown for comparison is the surface of the capillary-gravity wave when the shear layer is absent. The oscillations of the surface are related to the existence of multiple solutions and bifurcations (Chen \& Saffman 1980). It can be seen that the oscillations are enhanced by the shear layer and local maxima and minima are created. Similar results were obtained for $\Delta=0.2$. The surface oscillations are not found for $T<0.1$ or $T>0.5$.

The dependence of $\left(u_{\mathrm{s}}\right)_{\text {erest }}$ on $T$ was investigated for $\Omega$ ranging from 1.0 to 4.0 and $h / \lambda$ between 0.026 and 0.04 for $\Delta=0.05$. In all cases $\left(u_{\mathrm{s}}\right)_{\text {crest }}$ decreased monotonically with $T$ away from zero, going from values of around -0.8 for $T=0$ to -1.4 for $T=2.0$ so that the tendency to break was reduced. There were some mild oscillations of $\left(u_{\mathrm{s}}\right)_{\text {crest }}$ for $T$ in the range 0.1 to 0.3 , again associated with the existence of bifurcations in this range.

In conclusion, we wish to comment that preliminary studies of waves propagating against the drift, i.e. with negative phase speeds, indicated the non-existence of real solutions for ranges of the parameters. This could be traced to a coalescence of the two smaller wave speeds, and the appearance of complex wave speeds. A similar phenomenon was shown to occur in the dynamics of interfacial waves in the presence of a current (Saffman \& Yuen 1982), and it was pointed out that the non-existence can be identified with a hydrodynamic instability of the flow; in that case it is the 

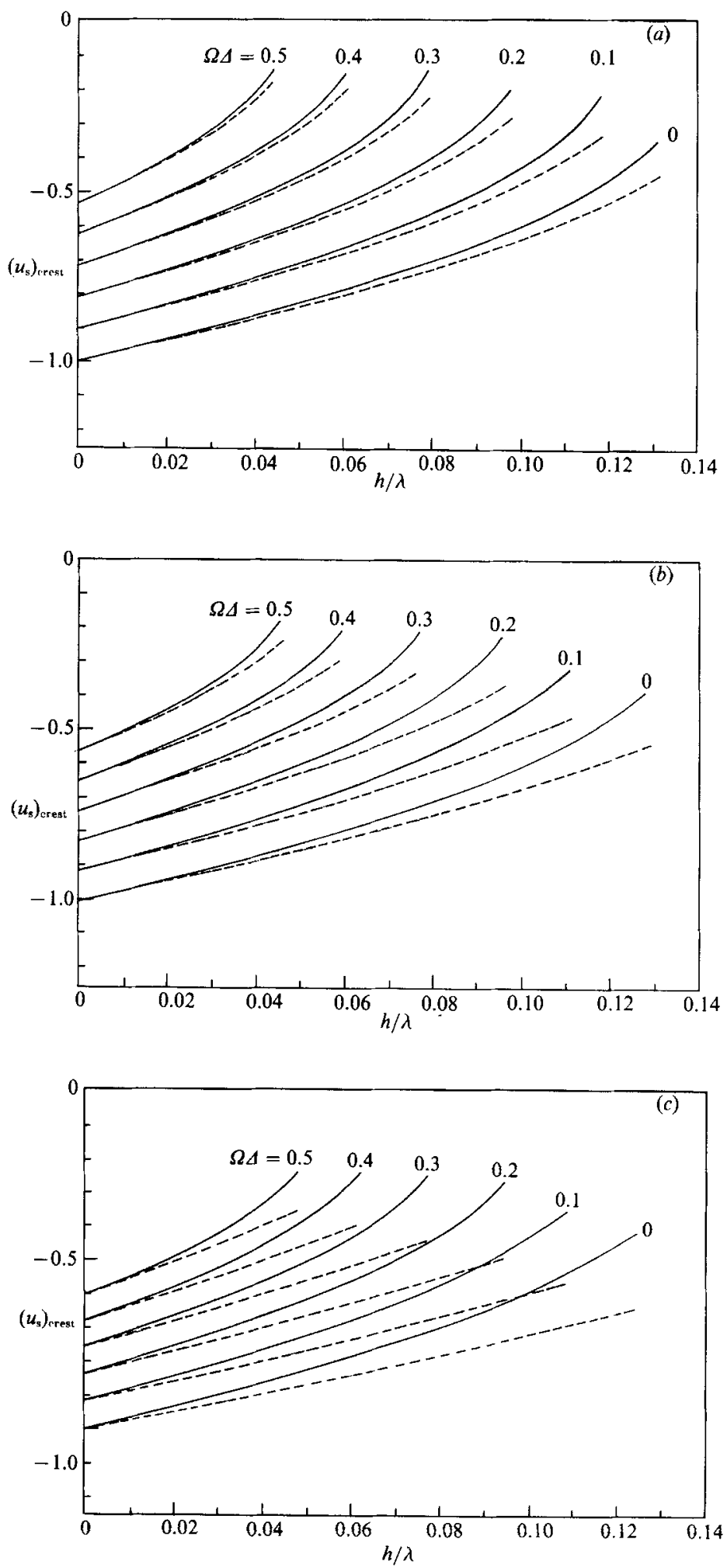

Fiqure 2. For caption see facing page. 


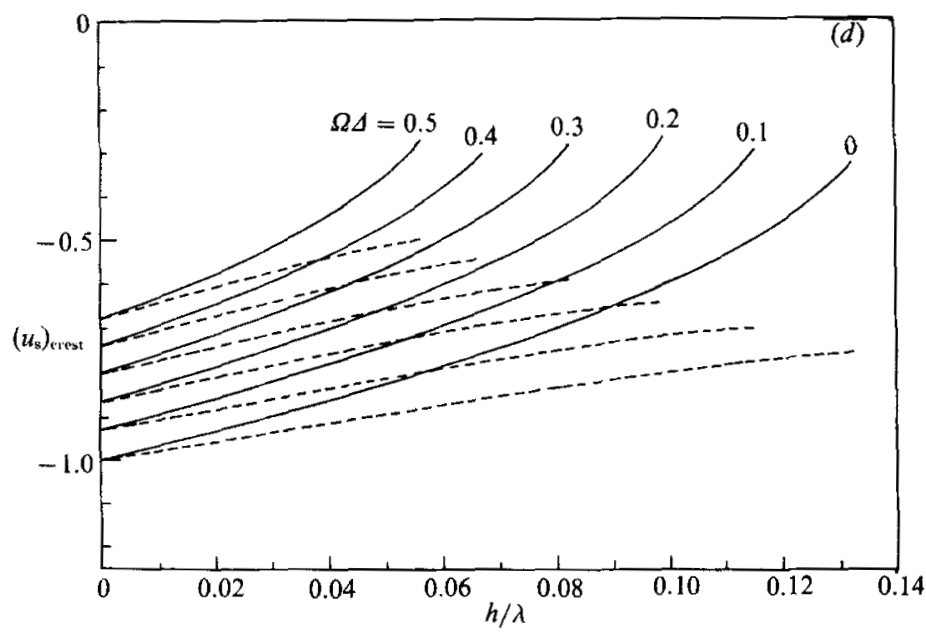

Figure 2. Variation of $\left(u_{\mathrm{s}}\right)_{\text {crest }} v s . h / \lambda$ for various drift speeds and layer thicknesses, with $T=0 .(a) \Delta=0.05,(b) 0.1,(c) 0.2,(d) 0.4$. Present computations, solid line. BP, dashed line.

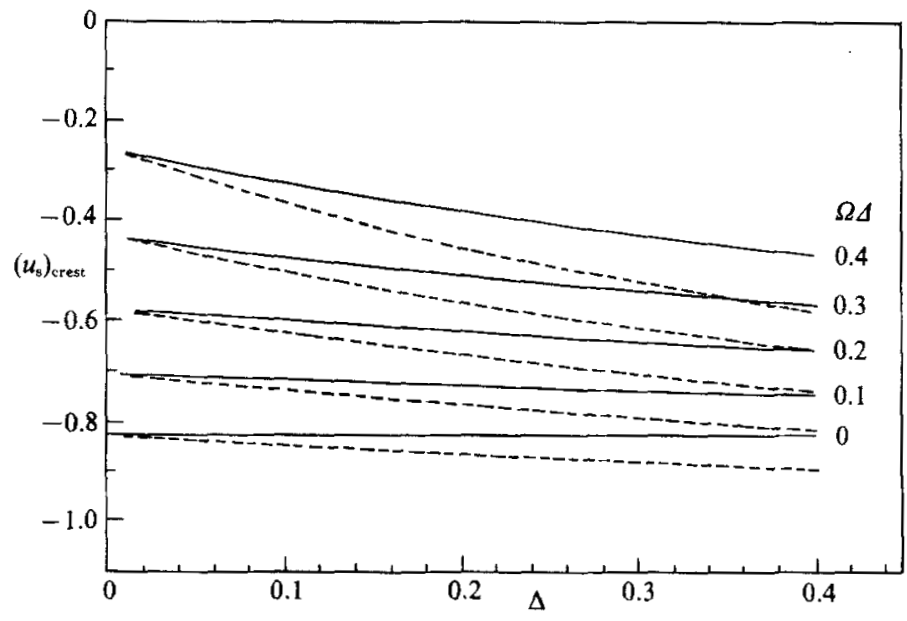

Figure 3. Dependence of $\left(u_{\mathrm{s}}\right)_{\mathrm{crest}}$ on $\Delta$ for $h / \lambda=0.05$ and various $\Omega \Delta$. Present computations, solid line. $\mathrm{BP}$, dashed line.

Kelvin-Helmholtz instability. There is likewise an instability of the drift layer for a range of the parameters. A study of this instability and its relevance to the generation of waves by wind will be described in subsequent work (Caponi, Yuen, Milinazzo \& Saffman, in preparation).

This work was supported by the Office of Naval Research (Grant N00014-89-J1164). We wish to thank Dr H. C. Yuen for valuable comments. We also wish to thank Dr L. Moorland for checking the results for capillary-gravity waves and assistance with figure 5 . 


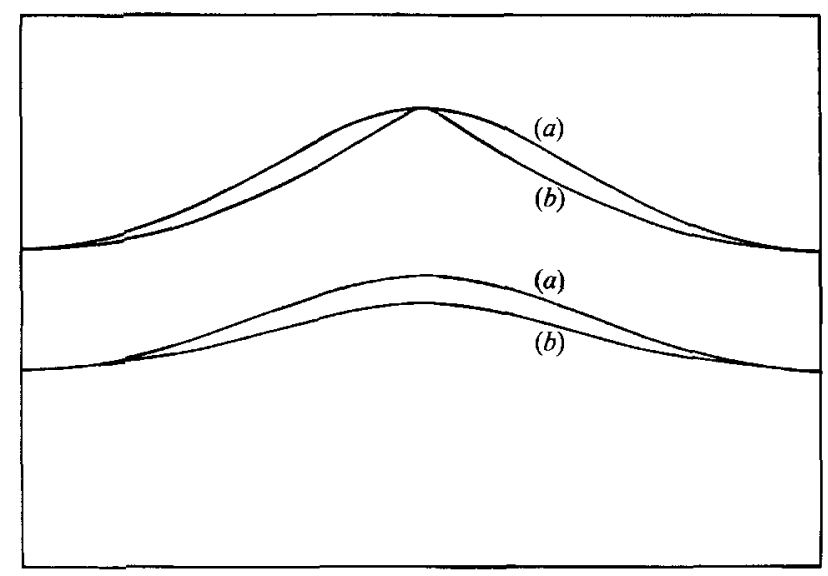

Figure 4. Shape of a gravity wave and the interface with and without drift layer for $h / \lambda=0.062$, $\Delta=0.4, \Omega A=0.456$. $(a)$ No shear layer, $(b)$ shear layer. Upper curves are the free surface; lower curve $(b)$ is the bottom of the shear layer and lower curve $(a)$ is a streamline.

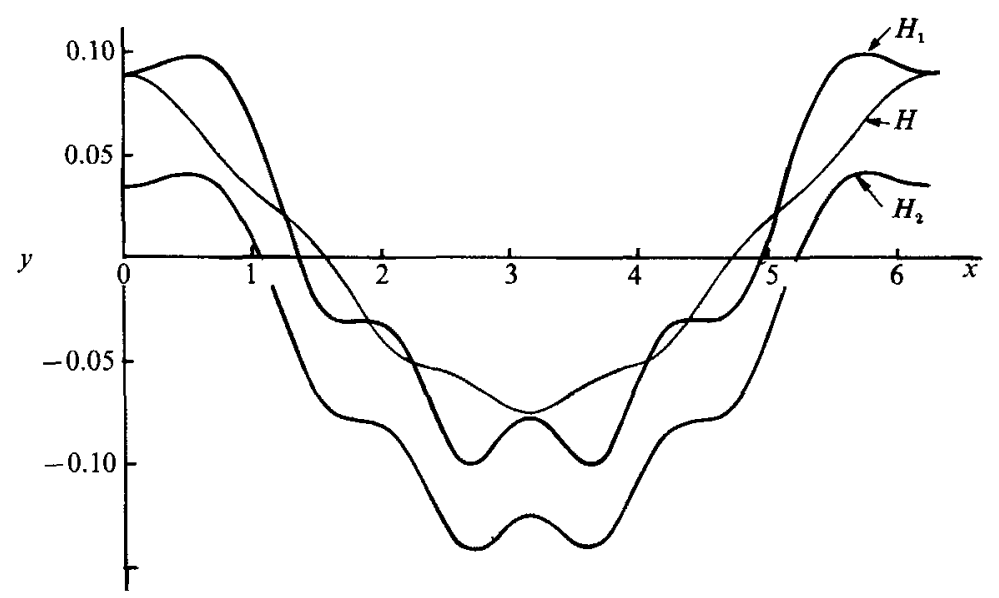

Figure 5. Heavy lines show shape of a capillary gravity wave and the bottom of the shear layer for $T=0.2, \Omega=1.0, \Delta=0.05$ and $h / \lambda=0.026$. Light line is surface when shear layer is absent.

\section{Appendix. The Levi-Cività relation}

Consider the irrotational fluid in the region $A B C D$ (see figure 1) between $y=H_{1}$ and $y=-h_{\infty}$, where $h_{\infty}$ is so large that the flow can be considered uniform at this depth and is moving with speed $c$ in a wave-fixed reference frame. Applying conservation of momentum to this region, we obtain

$$
p_{\infty}=g h_{\infty},
$$

were we have taken the pressure in the air to be zero. Note that (A 1) holds if surface tension is present.

From Bernoulli's equation in the irrotational flow,

we obtain using (A 1)

$$
p+\frac{1}{2}|\nabla \psi|^{2}+g y=B_{2},
$$


Applying momentum balance to the irrotational fluid in $A^{\prime} B^{\prime} C^{\prime} D^{\prime}$ between the lower surface $y=H_{2}$ and $y=-h_{\infty}$, we obtain

$$
g \lambda \Delta=\int_{H_{2}} p_{H_{2}} \mathrm{~d} x .
$$

The average of (A 2) along the interface $y=H_{2}$ gives

$$
B_{2}=\frac{1}{2 \lambda} \int_{H_{2}}|\nabla \psi|^{2} \mathrm{~d} x .
$$

Let $u$ denote the speed of the fluid on the lower interface at the mean depth where $y=-\Lambda$. From (A 2) and (A 3),

$$
c^{2}-u^{2}=2(p+g y)_{y=-\Delta}
$$

The Levi-Cività relation requires the vanishing of the right-hand side of (A 6), or equivalently from (A 5) that the average speed on the lower interface is the speed at its mean level. There is no obvious reason why these conditions should be satisfied exactly, although they may, depending upon circumstances, be a good approximation.

\section{REFERENCES}

Banner, M. L. \& Phillips, O. M. 1974 On the incipient breaking of small scale waves. J. Fluid Mech. 65, 647-656 (referred to herein as BP).

Brondbent, E. G. \& Moore, D. W. 1985 Waves of extreme form on a layer of uniform vorticity. Phys. Fluids 28, 1561-1563.

Chen, B. \& Saffman, P. G. 1980 Steady gravity-capillary waves on deep water. II Numerical results for finite amplitude. Stud. Appl. Maths 62, 95-111.

Dalrymple, R. A. 1974 A finite amplitude wave on a linear shear current. J. Geophys. Res. 79, 4498-4504.

Moore, D. W. \& Saffman, P. G. 1982 Finite amplitude waves in inviscid shear flow. Proc. R. Soc. Lond. A 382, 389-410.

Oramura, M. \& Oikawa, M. 1989 The linear stability of finite amplitude surface waves on a linear shearing flow. J. Phys. Soc. Japan 58, 2386-2396.

Saffman, P. G. \& Yuen, H. C. 1982 Finite-amplitude interfacial waves in the presence of a current. J. Fluid Mech. 123, 459-476.

Simmen, J. A. \& Safmin, P. G. 1985 Steady deep water waves on a linear shear current. Stud. Appl. Maths 73, 35-57.

Teles da Silva, A. F. \& Peregrine, D. H. 1988 Steep, steady surface waves on water of finite depth with constant vorticity. J. Fluid Mech. 195, 281-302.

ZufiriA, J. A. 1987 Non-symmetric gravity waves on water of infinite depth. J. Fluid Mech. 181, 17-39. 